\title{
Information Technology Tools Employed in Infection Control
}

\author{
Tamás Haidegger ${ }^{1}$, Viktor Varga ${ }^{2}$, Ákos Lehotsky $^{3}$, Péter Róna ${ }^{2}$, Róbert Pethes ${ }^{4}$, Péter Szerémy ${ }^{2}$, \\ László Szilágyi ${ }^{5}$, Tamás Ferenci ${ }^{4}$ and Levente Kovács ${ }^{4}$ \\ ${ }^{1}$ Óbuda University, Antal Bejczy Center for Intelligent Robotics, Budapest, Hungary \\ ${ }^{2}$ Clariton Ltd., Budapest, Hungary \\ ${ }^{3}$ Dept. of Surgical Research and Techniques, Semmelweis University, Budapest, Hungary \\ ${ }^{4}$ Óbuda University, John von Neumann Faculty of Informatics, Budapest, Hungary \\ ${ }^{5}$ Dept. of Electrical Engineering, Sapientia University of Transylvania, Tîrgu Mureș, Romania
}

e-mail: haidegger@irob.uni-obuda.hu,wolkje8@gmail.com, akos.lehotsky@gmail.com,rona.peter@gmail.com,pethes.robert@gmail.com, odonka@gmail.com, lalo@ms.sapientia.ro, ferenci.tamas@nik.uni-obuda.hu, kovacs.levente@nik.uni-obuda.hu

\begin{abstract}
Hygiene, in general is a key attribute to maintain safety and healthy procedures at various industries, ranging from food processing to medical care. It is linked to human behavior at multiple levels, which means, relevant standards and protocols are often bypassed due to the lack of competence, skill and willingness for compliance. The act of hand hygieneor hand rubbing, when performed with an alcoholic disinfectant solution-has become a standard practice both in personal hygiene and in the medical and clean site manufacturing domains. However, fundamental problems remain associated with the improper execution of hand hygiene (hand washing and rubbing) that calls for a technology-advanced solution. Technology-induced behavioral change is a recent emerging paradigm, where ICT tools properly integrated to the daily workflow of humans can significantly alter their routine behavior. The trend is now clearly visible in many domains of health care, and this paper aims to provide a general overview of the cutting edge technological solutions addressing the urging need of infection control.
\end{abstract}

Keywords - hand disinfection, objective evaluation of hand rubbing, hand hygiene control

\section{INTRODUCTION}

Healthcare-Associated Infections (HAI) are one of the most serious healthcare issues in the Western world, the $4^{\text {th }}$ leading cause of death in the USA [1], as even gloves may only reduce contamination by $70-80 \%$ [2]. Due to the rise of antibiotic resistant stems, such as Methicillin-Resistant Staphylococcus Aureus (MRSA), Clostridium difficile, Drug-resistant Streptococcus pneumonia infections remain a lethal threat [3]. An earlier publication from a UK hospital showed that "After contact with patient's environment, $10 \%$ of Health Care Workers (HCW) tested positive for MRSA on their fingertips, of whom $41 \%$ had used hand rubs" [4]. Eradicating HAI is an unmet medical/humanitarian need, and the lack of success in controlling it so far underlines that it requires very complex and integrated solution to tackle the problem. Recent studies clearly showed that training and education only cannot solve this problem, advanced tools must be employed [5].

According to the World Health Organization's (WHO) estimates, HAI results in $1.4 \mathrm{~m}$ cases a day globally, leading to a quarter of a million unnecessary deaths a year in the developed countries alone [6]. In the USA, according to the Centers for Disease Control and Prevention (CDC), there are more than 2 million infections [7] and more than 99,000 infection-related deaths each year [8]. If HAI occurs, it extends the hospital stay by an average 16 days, and the mortality rates increase to 5 times higher [9].

Despite all the previous efforts-including governmental campaigns and global educational programs-Western hospitals still have a $10 \%$ average HAI ratio [6]. Newest US reports show a gradual decrease in overall hospital-acquired complications, yet the numbers are still over $13 \%$ [10]. Hospital Infections Disclosure Act (HIDA) HIDA was implemented in 2007 and currently 24 states have adopted legislation requiring public reporting of hospital infection rates and 19 have adopted legislation regarding the reporting of hospital infection rates. Regular hand washing has already been proved through numerous peer reviewed studies to be efficient to reduce the incidence of diseases, thus improving the quality of life [11].

\section{THE COST OF POOR HAND HYGIENE}

Costs for HAI in Europe are approximately $\$ 9.3$ billion annually [12]. The most common type, central lineassociated bloodstream infections were found to be the most costly HAIs at over $\$ 45 \mathrm{k}$ extra cost per incidence [13]. Pennsylvania State in the US reported a total of 11,500 infections in 2005 that accounted for over $\$ 2$ bn extra costs [14]. HAI is estimated to cost $\$ 96 \mathrm{bn}-\$ 147 \mathrm{bn}$ each year to the US economy [15]. It is estimated that in the EU an average 500 bed hospital loses $\$ 1.87 \mathrm{~m}$ on infections annually [16].

According to a very conservative WHO estimation, effective hand disinfection has shown to decrease HAI by at 
least $30 \%$, which may manifest in an annual saving of $€ 18 \mathrm{bn}$ in the Western world. Up to $66 \%$ of certain types of HAI are caused by improper hand hygiene [17]. A recent survey found that reported direct cost savings on avoided HAI cases accounted for hand hygiene ranged from $\$ 32 \mathrm{~K}-476 \mathrm{~K}$ for various hospitals [18].

Compliance of the medical staff to hand hygiene is only $60 \%$ even at well-reputed institutions, while the average reported incidence of nosocomial infections is $7.1 \%$ in the Western world. Governments and insurance companies are now explicitly charging the hospitals for the avoidable costs of HAI. Since Oct. 1, 2008, the U.S. Centers for Medicare and Medicaid Services no longer provide reimbursement for cases with a diagnosis of HAI [19]. Further, in the UK, NHS Quality Premium payments to Clinical Commissioning Groups (CCGs) are linked to HAI prevention efforts $(12.5 \%$ of the premium in 2013-14) [20].

\section{BEST PRACTICES IN HAND HYGIENE FOR INFECTION} CONTROL

Alcohol-based hand rubs are recognized as the most effective tools to prevent HAI [21], therefore the use of them became a general standard and a European Norm [22]. The same methods apply to the biotech and clean industry, enforced by the Good Manufacturing Practice (GMP) [23].

First, the WHO defined the "Five Moments for Hand Hygiene" as part of the WHO Guidelines on Hand Hygiene in Health Care to add value to any hand hygiene improvement strategy. It defines the key moments for hand hygiene (compliance), overcoming misleading language and complicated descriptions, presenting a unified vision and promoting a strong sense of ownership of HCWs [24].

The WHO six-step hand washing procedure (Figure 1) is the globally recognized protocol for clean hands [25]. It can be found in many official recommendations and guidelines. By applying this protocol, it becomes easier to remember cleaning the whole hand surface efficiently, yet only in $8 \%$ of the cases the protocol is followed thoroughly [26]. Moreover, $27 \%$ of people fail to achieve a complete coverage of their hands even when they perform the 6-steps, leading to potential infections [27]. Some groups therefore promote the "Self-responsible application of hand rubs", trusting HWCs to cover the most critical areas [28].

Rigorous education and promotion campaign at the National University Health System Singapore resulted in the growth of compliance in their intensive care units from $38 \%$ to $69 \%$ in 3 years, while MRSA acquisition ratio dropped from $10.1 \%$ to $1.1 \%$ [29]. Another recent report from Vanderbilt University revealed that over 5 years of continuous education and training, average compliance ratio (only considering before/after patient) increased from $58 \%$ to
$96.6 \%$, while urinary tract infections dropped by $33 \%$, pneumonia-linked ventilation infections by $61 \%$ and centralline associates bloodstream infections by $80 \%$, respectively [30]. This data show the difficulty to implement correct hand hygiene practices in a busy environment, where human factors play a huge role.
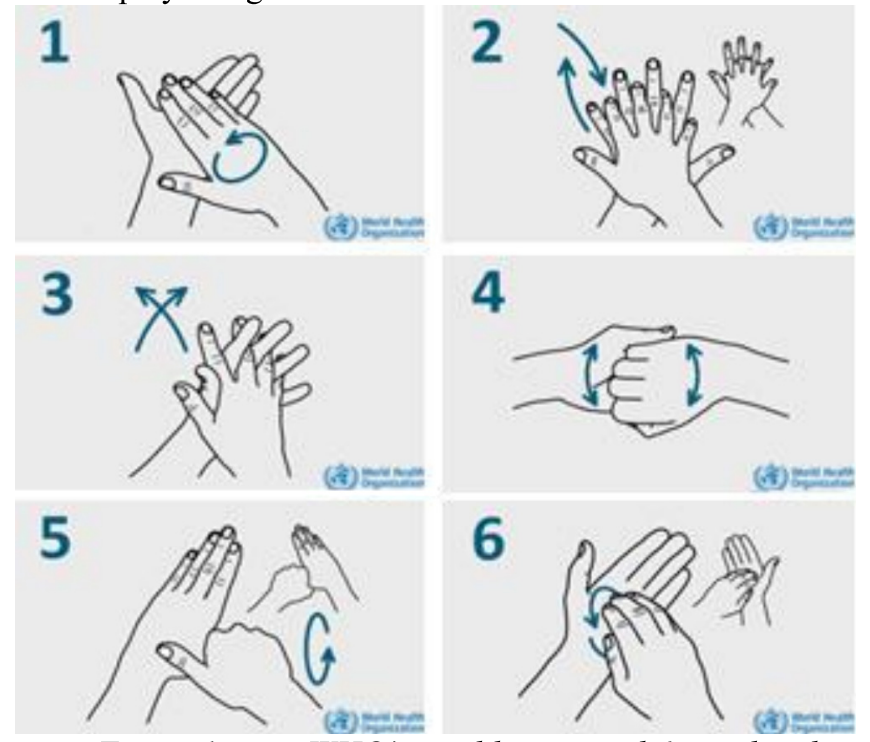

Figure $1 \quad$ WHO's world renowned 6-step hand

hygiene protocol - a global standard.

Source: WHO, http://www.who.int/gpsc/en/

The two most important performance indicators of hand hygiene are monitoring of the event compliance and the technique. In other words, it has to be monitored that:

1) employees always rub their hands when it is recommended by policy: event compliance;

2) they always do it the right way: technique.

Traditionally, hand hygiene compliance - both event and technique - has been assessed by direct human observation method (current WHO recommendation). It has never been considered to be statistically trusted [31], and has serious drawbacks:

1) observer-distortion (Hawthorne) effect [32]—people tend to behave better if they are visually controlled,

2) high cost,

3) timeliness,

4) subjectivity of the observer,

5) poor statistical power due to the low sample size.

\section{HAND HYGIENE CONTROL AND MONITORING}

HCW's adherence to hand hygiene traditionally has been well below the required level. In the past 10 years, several 
commercial products and approaches have been developed to required between compliance monitoring and education of the technique. Many companies started to develop ICT tools to support HCWs, having realized the importance of hand hygiene linked to HAI.

1) Software solutions exist to support the registration of hand washing events, however, these require manual data input.

2) Automated compliance monitoring systems have been developed, tracking the spatial movement of the staff and linking it to hand hygiene events.

3) There are educational tools using UV-reflective gels to demonstrate hand rub use through removing the reflective material. Occasionally, hand washing quality is checked under UV lighting, but only verified by the naked eye.

4) Newest technologies address the issue of objective monitoring and assessment of the hand rubbing technique.

Event compliance monitoring

- Automated: Biovigil, HillRom, Hyginex, IntelligentM, GeneralSensing MedSense, Safe-Hands, SwipeSense, Xhale Hygreen

- Direct observation: by infection control nurses

Complementary

Technique compliance monitoring

- Automated (indirect performance measures): SureWash

- Automated (direct performance measures): Hand-in-Scan

- Direct observation: by infection control nurses with or

Figure 2 Technology segments of electronic hand increase it. It is generally accepted that a healthy balance is without UV-lamp control hygiene control and support.

\section{A. Automated (electronic) observation:}

Technology is coming to the rescue with computerized performance indicators for measuring improvement in HCWs' adherence to hand hygiene protocols. Classically, only the overall hand rub consumption is monitored. From 2010 and on, hand hygiene solution manufacturers in healthcare have grown tremendously. Several developed their own hand hygiene monitoring system, or training courses based on the WHO's guidelines and CDC recommendation [33]. Many of these products are based on the concept of monitoring the "Point of Care" (PoC) for every patient, meaning that an electronic sensor is placed in the wardroom, which communicates with the worker's badge in order to remind them to wash hands when entering or leaving the patient's surrounding (Fig. 3). Electronic surveillance has many options; the key feature is to be able to automatically identify the location and the motion of the HWC relative to the POC. This can be achieved employing:

- RFID tags

- US identification

- RF tracking

- Video monitoring.

Table 1. summarizes most of these systems identified (solutions publicly known as of now), along with their major features. The various features enable different level of technology-integration. Automated data collection and database recording are considered to be the most important features of these systems.

\section{i) Event compliance monitoring}

It is not the mere number of hand washing events per day ("frequency") that is only important but also when (and how) $\mathrm{HCW}$ wash their hands. The number of opportunities to perform hand hygiene (as determined by WHO's 5-moments a

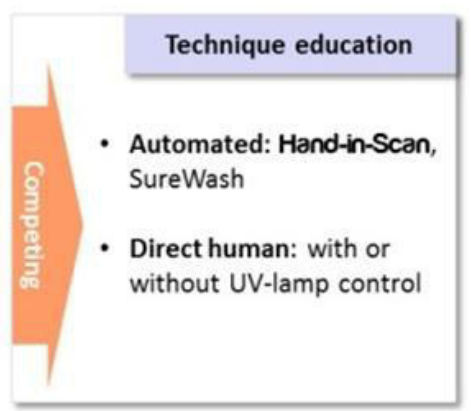

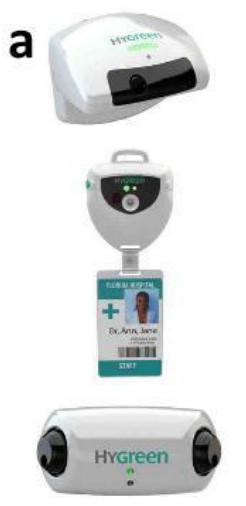
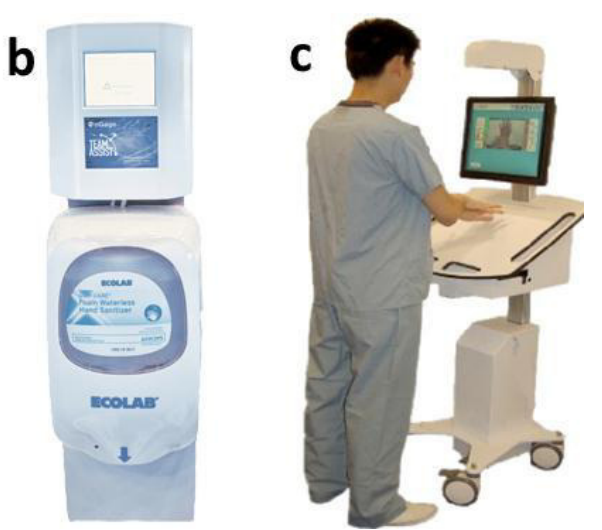

Figure 3 Examples of electronic hand hygiene systems: HyGreen, Proventix nGage and SureWash. 
and healthcare-facility policies and the number of coinciding events that occur) is the foundational element to be measured, yet many devices focus on a more simple approach, e.g., POC on/out measurement [32]. It has been reported that equipping one patient care using with electronic sensors for consumption monitoring may cost up to $\$ 40,000$ [34]. These monitoring systems, such as IntelligentM (www.higenx.com/), HigenX (www.higenx.com), Dubmed (www.newswiretoday.com/ /news/141062), i360hygiene (www.i360hygiene.com) or HandGiene (www.hpnonline.com/inside/2014-03/1403-IPHHSurveillance.html) have the ability to record the hand hygiene events, monitoring only compliance and not the quality. Studies showed that raising $\mathrm{HH}$ compliance among healthcare workers results in a $10-80 \%$ decrease in HAI cases. For instance, using Ecolab's Proventix nGage (http://proventix.com/ngage) system for a 7-month trial period, the Princeton Baptist Medical Center of Birmingham reported a decrease of 159 patient days and reduced hospital net losses of over $\$ 133,386$, reflected on just 2,652 patient admissions [34]. A more sophisticated system has been developed by using alcohol sensors. The US based company's products HyGreen (hygreen.com) and Biovigil (www.biovigilsystems.com) have extended the PoC concept: a wall-mounted sensor or a badge has to sense alcohol prior to entering a patient's area, which is isolated with a proximity sensor located on the top of the bed, or at the doorway. If alcohol is not present when indicated, the worker's badge vibrates, as a reminder function. However, this system only detects the presence of alcohol but not the quality of the hand wash. Recent reports revealed that after 6 months of installment, the usage of these monitoring technologies is extremely low, therefore smarter workflow integration methods are required.

\section{ii) Technique monitoring}

Most companies focus on indirect performance measures, like hand wash duration or assessment via direct video monitoring [30]. Technologies that enable this direct observation and try to make it more "objective" are e.g., the handheld UV lamps used with UV-dyed hand rub. These have been on the market for more than 30 years, but have not made any revolutionary effect in hand hygiene, since they could not solve the other inherent problems of direct observation (Hawthorne effect, problem of the power-gap, etc.). Companies in this field are: Glow Germ (http://handhygiene.com), Anios http://www.securimed.fr/ /caissonpedagogique-9836.html), Dermalux (www.disinfection.ch/ /hygieneschulungen/ /hygieneschulungen.htm), AllPax Derma LiteCheck Box (www.allpax.de/product_info.php /info/p9620_Derma-LiteCheck--Box.html).
SureWash (Glanta Ltd, IR) was the first product on the market using video stream to evaluate hand washing performance. Its primary focus is educational use, the system helps the user performing the WHO 6-step procedure while measuring the dry-run performance (no hand rub used) with real-time video recording and stream processing. The system is overviewed by the infection control specialist, and can be placed into various departments of the hospital for periodic training.

Hand-in-Scan (HandInScan Kft.) is a recent innovation of a Hungarian start-up company, employing digital imaging and image processing to instantaneously identify any missed regions on the hands. Hand-in-Scan objectively evaluates and records every hand disinfection event per person, and is able to generate statistics and proper reporting to the management (Fig. 4). Implementing the experiential learning theory, the user learns the correct hand washing technique while performing it in front of the device. Hand-in-Scan provides immediate feedback on hand washing performance, resulting in healthcare worker's hand hygiene compliance and technique improvement. The system eliminates the blamefactor, and helps to avoid the boss-subordinate conflict [35].

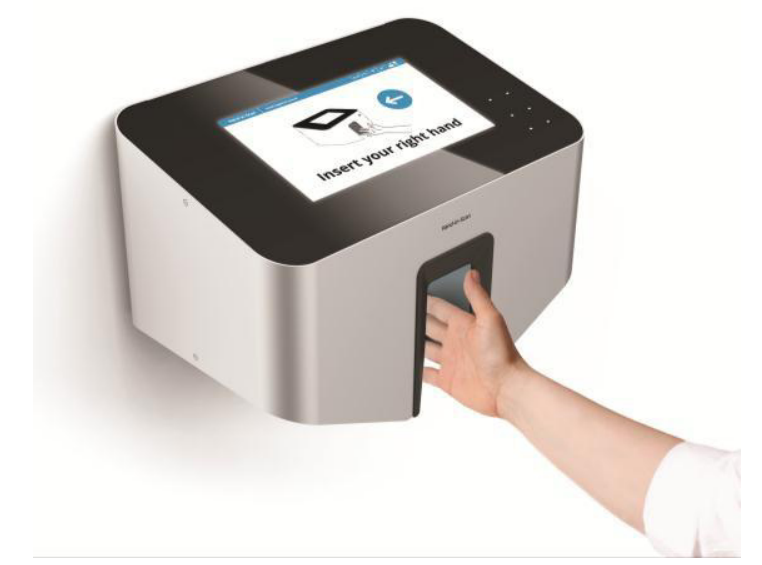

Figure 4 The Hand-in-Scan Medical Trainer hand hygiene monitoring and control system.

\section{Discussion}

An ideal hand hygiene efficiency system should contain both event compliance and technique monitoring. In this sense, the field is really in its infancy yet. Almost all monitoring systems listed above primarily and only focus on event compliance, checking on the occurrence and frequency of hand rubbing, but not the quality. Understandably, if the alcoholic solution is not applied correctly, its effectiveness remains low. There are only two companies approaching the problem with more direct (hand washing technique) 
performance measurement, the SureWash educational system and Hand-in-Scan.

All these systems have great importance in the future of infection control, since they generate digital data, and make measurable aspects of hand hygiene impossible to adequately monitor before. These new databases enable researchers to draw a better profile on infections, how they spread, transmit and carry on, while the systems behind are emphasizing the importance of self-responsibility of HCW. Technology does induce behavioral change.

\section{vi. Conclusions}

Traditional, visual observation of the health care workers requires extensive human resources and is not effective. There is a global trend to develop hand hygiene control systems with different approaches, to foster improvement in safe care. The paper identified the main technological directions, systems and concepts for hand sterilization and hand sterilization checking. The list includes all companies spotted, currently selling or developing a solution for hand hygiene control. This field deserves attention from the biomedical engineering community, since the emerging electronic/automated platforms open space for evidencebased medicine in infection control as well.

\section{ACKNOWLEDGMENT}

T. Haidegger is a Bolyai Fellow of the Hungarian Academy of Sciences. This work has been supported by ACMIT (Austrian Center for Medical Innovation and Technology), which is funded within the scope of the COMET (Competence Centers for Excellent Technologies) program of the Austrian Government.

\section{REFERENCES}

[1] General Electric Co., "HAI: The unknown killer", available at: https://www.pinterest.com/pin/179229260142509135/

[2] Centers for Disease Control and Prevention, "Hand hygiene guidelines fact sheet," 2002, available at: http://www.cdc.gov/media/pressrel/ fs $021025 . h$ tm

[3] A. Huttner, S. Harbarth, J. Carlet, S. Cosgrove, H. Goossens, A. Holmes, et al., "Antimicrobial resistance: a global view from the 2013 World Healthcare-Associated Infections Forum," Antimicrob Resist Infect Control, vol. 2, paper 31, November 2013.

[4] M.L. McLaws, "The relationship between hand hygiene and health care-associated infection: it's complicated," Infect Drug Resist, vol. 8, pp. 7-18, 2015.

[5] S.P. Jayaraman, M. Klompas, M. Bascom, X. Liu, R. Piszcz, S.O. Rogers jr, and R. Askari, "Hand-hygiene compliance does not predict rates of resistant infections in critically ill surgical patients," Surg Infect, vol. 15 , no. 5, pp. 533-539, 2014.
[6] WHO guidelines on hand hygiene in health care. Geneva: World Health Organization, 2009

[7] Centers for Disease Control and Prevention, "Antibiotic resistance threats in the United States", available at: http://www.cdc.gov/ drugresistance/threat-report-2013/

[8] Scott, R. D. "The direct medical costs of healthcare-associated infections in US hospitals and the benefits of prevention," pp. 1-16, March 2009, available at: http://www.cdc.gov/HAI/pdfs/hai/Scott_ CostPaper.pdf

[9] Pennsylvania Health Care Cost Containment Council, "The impact of Healthcare-Associated Infections (HAI) in Pennsylvania Hospitals 2010", pp. 1-22, February 2012, available at: http://www.phc4.org/ reports/hai/10/docs/hai2010report.pdf

[10] U.S. Dept. of Health \& Human Services, "New HHS Data Shows Major Strides Made in Patient Safety, Leading to Improved Care and Savings," 7 May 2014, available at: http://inoovations.cms.gov/Files/ reports/patient-safety-results.pdf

[11] Association for Professionals in Infection Control and Epidemiology, Guide to Hand Hygiene Programs for Infection Prevention, 24 June 2015, available at: http://www.apic.org/Professional-Practice/ Implementation-guides

[12] HLC092C - Healthcare-Acquired Infection: Devices, Pharmaceuticals, and Environmental Products, BCC Report, August 2011, available at: http://www.bbcresearch.com/market-research/healthcare/ healthcare-acquired-infections-hlc092a.html

[13] E. Zimlichman, D. Henderson, O. Tamir, C. Franz, P. Song, C.K. Yamin, C. Keohane, C.R. Denham, D.W. Bates, "Health careassociated infections: a meta-analysis of costs and financial impact on the US health care system," JAMA Intern Med, vol. 173, no. 22, pp. 2039-2046, 2013

[14] The cost of infection. The economic argument for infection prevention, available at: http://www.hospitalinfection.org/cost-of-infection.shtml

[15] A. Marchetti, and R. Rossiter, "Economic burden of healthcareassociated infection in US acute care hospitals: societal perspective," $J$ Med Econ, vol. 16, no. 12, pp. 1399-1404, December 2013

[16] Eurostat, "Health care expenditure by financing agent", 11 May 2015, available at: $h t t p: / / a p p s s o . e u r o s t a t . e c . e u r o p a . e u / n u i / s h o w . d o ? d a t a s e t=$ hlth_sha_hf

[17] C.A. Umscheid, M.D. Mitchell, J.A. Doshi, R. Agarwal, K. Williams, and P.J. Brennan, "Estimating the proportion of healthcare-associated infections that are reasonably preventable and the related mortality and costs", Infect Control Hosp Epidemiol, vol. 32, no. 2, pp. 101-114, 2011

[18] Centers for Medicare \& Medicaid Services, "Hospital-acquired conditions", September 2014, available at: http://www.cms.gov/Medicare/ Medicare-Fee-for-Service-Payment/HospitalAcqCond/ index.html

[19] NHS England, "Quality Premium: 2013/14 guidance for CCGs", March 2013, available at: http://www.england.nhs.uk/wp-content/ uploads/2013/05/qual-premium.pdf

[20] D. Pittet, and J.M. Boyce, "Hand hygiene and patient care: pursuing the Semmelweis legacy," Lancet Infect Dis vol. 1, pp. 9-20, April 2001

[21] EN 1500:2013 Chemical disinfectants and antiseptics-Hygienic handrub-Test method and requirements, May 2013, available at: http://shop.bsigroup.com

[22] S. Sutton, "Hand Washing, Hygiene, CGMP, and Science," J GXP Compliance, vol. 14, no. 1, pp.62-69, 2010

[23] World Health Organization, "Five moments for hand hygiene", available at: www.who.int/gpsc/tools/Five moments/en

[24] World Health Organization, "The evidence for clean hands", http://www.who.int/gpsc/country work/en/

[25] H.Y. Park, S.K. Kim, Y.J. Lim, S.H. Kwak, M.J. Hong, H.M. Mun, S.Y. Park, H.J. Kim, H.R. Choi, J.S. Jeong, and .H.Choi, "Assessment of the appropriateness of hand surface coverage for health care workers according to World Health Organization hand hygiene guidelines," Am $J$ Infect Control, vol. 42, no. 5, pp. 559-561, May 2014 
[26] L. Szilágyi, T. Haidegger, Á. Lehotsky, M. Nagy, E.A. Csonka, X.Y. Sun, KL Ooi, and D. Fisher, "A large-scale assessment of hand hygiene quality and the effectiveness of the "WHO 6-steps"," BMC Infect Dis vol 13, paper 249, May 2013

[27] G. Kampf, M. Reichel, Y. Feil, S. Eggerstedt, and P.M. Kaulfers, "Influence of the rub-intechnique on required time and hand coverage in hygienic hand disinfection," BMC Infect Dis, vol. 8, paper 149, August 2008

[28] D. Fisher, "Hand hygiene and its role in bringing about MRSA control in Singapore. Save lives, clean hands," in: Hand Hygiene International Conference, Taiwan, 2011

[29] C. Kalb, "How a team of doctors at one hospital boosted hand washing, cut infections and created a culture of safety", Yahoo News, 21 July 2014, available at: http://news.yahoo.com/clean-hands--vanderbilt-shand-washing-initiative-172312795.html

[30] K.M. Pyrek, "Hand hygiene monitoring goes high-tech," Infection Control Today, 3 February 2012, available at: http://www.infectioncontroltoday.com/articles/2012/02/hand-hygienemonitoring-goes-hightech.aspx
[31] J.A. Srigley, C.D. Furness, G.R. Baker, and M. Gardam, "Quantification of the Hawthorne effect in hand hygiene compliance monitoring using an electronic monitoring system: a retrospective cohort study," BMJ Qual Saf, in press, doi:10.1136/bmjqs-2014-003080

[32] M.A. Ward, M.L. Schweizer, P.M. Polgreen, K. Gupta, H.S. Reisinger, and E.N. Perencevich, "Automated and electronically assisted hand hygiene monitoring systems: a systematic review," Am J Infect Control, vol. 42 , no. 5, pp. 472-478, May 2014

[33] L. Arnoldo, D. Pittet, J. Boyce, H. Sax, and B. Allegranzi. "O038: Automated/electronic systems for hand hygiene monitoring: a systematic review," Antimicrob Resist Infect Control, vol. 2, Suppl 1, paper O38, pp. 1, 2013

[34] S. Cantrell, "Electronic hand-hygiene surveillance systems: the new gold standard?", Healthcare Purchasing News, March 2014, available at: http://www.hpnonline.com/inside/2014-03/1403-IP-HHSurveillance $. \mathrm{html} /$

[35]Á. Lehotsky, L. Szilágyi, T. Ferenci, L. Kovács, R. Pethes, Gy. Wéber, and T. Haidegger, "Quantitative impact of direct, personal feedback on hand hygiene technique," J Hosp Infect, vol. 91, no. 1 pp. 81-84, 2015

Table 1. Complete list of hand hygiene monitoring and control systems.

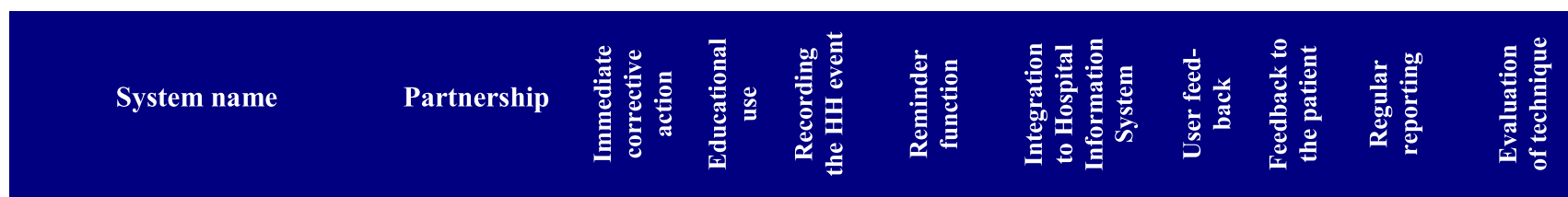

\begin{tabular}{|c|c|c|c|c|c|c|c|c|c|c|}
\hline 3M Clean-Trace & & $\mathrm{X}$ & $\mathrm{X}$ & & & & $\mathrm{X}$ & & & \\
\hline AccuTrack & & & $\mathrm{X}$ & $\mathrm{X}$ & & & $\mathrm{X}$ & & & \\
\hline ANIOS & & $\mathrm{X}$ & $\mathrm{X}$ & & & & $\mathrm{X}$ & & & \\
\hline Arista & & & & $\mathrm{X}$ & & & & & $\bar{X}$ & \\
\hline Arrowsight & & $\mathrm{X}$ & & $\mathrm{X}$ & & $\mathrm{X}$ & $\mathrm{X}$ & & & \\
\hline BioVigil monitoring & & $\mathrm{X}$ & & $\mathrm{X}$ & $\mathrm{X}$ & $\mathrm{X}$ & & $\mathrm{X}$ & & \\
\hline $\begin{array}{l}\text { Brevis GlitterBug Maxi View } \\
\text { Box }\end{array}$ & & $\mathrm{X}$ & $\mathrm{X}$ & & & & & $\mathrm{X}$ & & \\
\hline DaRo & & $\mathrm{X}$ & & & & & $\mathrm{X}$ & & & \\
\hline DEB DebMed GMS & & & & $\mathrm{X}$ & & & & & & \\
\hline Derma LiteCheck box & & $\mathrm{X}$ & $\mathrm{X}$ & & & & $\mathrm{X}$ & & & \\
\hline $\begin{array}{l}\text { Dermalux Box Checkbox } \\
\text { Multimedia }\end{array}$ & & $\mathrm{X}$ & $\mathrm{X}$ & & & & $\mathrm{X}$ & & & \\
\hline Ekahau Inc. HH Monitoring & & & & $\mathrm{X}$ & $\mathrm{X}$ & & & & & \\
\hline Ephygie Hand & & & & $\mathrm{X}$ & & $\mathrm{X}$ & & & $\bar{X}$ & \\
\hline Glo Germ prod. & & & $\mathrm{X}$ & & & & & & & \\
\hline Glowshop & & $\mathrm{X}$ & & & & & $\mathrm{X}$ & & & \\
\hline Glowtech & & $\mathrm{X}$ & $\mathrm{X}$ & & & & & $\mathrm{X}$ & & \\
\hline GOJO & $\begin{array}{l}\text { Hill-room, } \\
\text { Purell \& Provon }\end{array}$ & & & $\mathrm{X}$ & & $\mathrm{X}$ & & & monthly & \\
\hline hand giene corp & & $\mathrm{X}$ & $\mathrm{X}$ & $\mathrm{X}$ & $\mathrm{X}$ & $\mathrm{X}$ & & $\mathrm{X}$ & & \\
\hline Hand-in-Scan & & $\mathrm{X}$ & $\mathrm{X}$ & $\mathrm{X}$ & $\mathrm{X}$ & $\mathrm{X}$ & $\mathrm{X}$ & $\mathrm{X}$ & $\mathrm{X}$ & $\mathrm{X}$ \\
\hline Handy audit & & & & $\mathrm{X}$ & & & & & $\mathrm{X}$ & \\
\hline Hangenix & & $\mathrm{X}$ & & $\mathrm{X}$ & $\mathrm{X}$ & & & & $\mathrm{X}$ & \\
\hline HigenX & & & & $\mathrm{X}$ & $\mathrm{X}$ & & & & monthly & \\
\hline Hill-rom & GOJO, Ecolab & $\mathrm{X}$ & $\mathrm{X}$ & $\mathrm{X}$ & $\mathrm{X}$ & $\mathrm{X}$ & & & monthly & \\
\hline HygenX & & & & $\mathrm{X}$ & $\mathrm{X}$ & $\mathrm{X}$ & & & $\mathrm{X}$ & \\
\hline i360 hygiene & Surewash & & & $\mathrm{X}$ & $\mathrm{X}$ & & $\mathrm{X}$ & $\mathrm{X}$ & & \\
\hline IntelligentM & & & & $\mathrm{X}$ & $\mathrm{X}$ & $\mathrm{X}$ & & $\mathrm{X}$ & monthly & \\
\hline Proventix nGage & & & & $\mathrm{X}$ & $\mathrm{X}$ & $\mathrm{X}$ & & $\mathrm{X}$ & & \\
\hline Semmelweis Hand Hygienom. & & & & $\mathrm{X}$ & & & & & & \\
\hline Sterilogy LLC & & & & $\mathrm{X}$ & & & & $\mathrm{X}$ & $\mathrm{X}$ & \\
\hline Surewash & i360 hygiene & & $\mathrm{X}$ & & & & & & & $\mathrm{x}$ \\
\hline SwipeSense & & & & $\mathrm{X}$ & & & & $\mathrm{X}$ & $\mathrm{X}$ & \\
\hline Xhale Inc. Hygreen & & $\mathrm{X}$ & & $\mathrm{X}$ & $\mathrm{X}$ & $\mathrm{X}$ & $\mathrm{X}$ & $\mathrm{X}$ & & \\
\hline
\end{tabular}

